\title{
Nrf2 establishes a glutathione-mediated gradient of UVB cytoprotection in the epidermis
}

\author{
Matthias Schäfer, ${ }^{1,5}$ Sabine Dütsch, ${ }^{1}$ Ulrich auf dem Keller, ${ }^{1}$ Fatemeh Navid, ${ }^{2}$ Agatha Schwarz, ${ }^{2}$ \\ Delinda A. Johnson, ${ }^{3}$ Jeffrey A. Johnson, ${ }^{3}$ and Sabine Werner ${ }^{1,4}$ \\ ${ }^{1}$ Department of Biology, Institute of Cell Biology, ETH Zurich, CH-8093 Zurich, Switzerland; ${ }^{2}$ Department of Dermatology, \\ Christian-Albrechts-University of Kiel, D-24105 Kiel, Germany; ${ }^{3}$ University of Wisconsin, School of Pharmacy, Madison, \\ Wisconsin 53705, USA
}

\begin{abstract}
Ultraviolet (UV) B irradiation can severely damage the skin and even induce tumorigenesis. It exerts its effects by direct DNA modification and by formation of reactive oxygen species (ROS). We developed a strategy to genetically activate target gene expression of the transcription factor NF-E2-related factor 2 (Nrf2) in keratinocytes in vivo based on expression of a constitutively active Nrf2 mutant. Activation of Nrf2 target genes strongly reduced UVB cytotoxicity through enhancement of ROS detoxification. Remarkably, the protective effect was extended to neighboring cells. Using different combinations of genetically modified mice, we demonstrate that Nrf2 activates the production, recycling, and release of glutathione and cysteine by suprabasal keratinocytes, resulting in protection of basal cells in a paracrine, glutathione/cysteine-dependent manner. Most importantly, we found that endogenous Nrf2 controls selective protection of suprabasal keratinocytes from UVB-induced apoptosis through activation of cytoprotective genes. This finding explains the preferential UVB-induced apoptosis of basal cells, which is important for elimination of mutated stem cells as well as for preservation of skin integrity. Taken together, our results identify Nrf2 as a key regulator in the UV response of the skin.
\end{abstract}

[Keywords: Apoptosis; Nrf2; UVB; reactive oxygen species; glutathione]

Supplemental material is available at http://www.genesdev.org.

Received November 18, 2009; revised version accepted March 30, 2010.

Ultraviolet (UV) B irradiation is one of the most dangerous insults for the skin, and causes sunburn, erythema, and photoaging. Most importantly, UV-induced DNA damage is a major risk factor for skin carcinogenesis. The highly energetic short-wavelength UVB light (280$320 \mathrm{~nm}$ ) has the strongest carcinogenic potency (Bowden 2004). UVB damages DNA directly, resulting in formation of dimeric photoproducts, and indirectly by reactive oxygen species (ROS) formation, causing strand breaks and hydroxylation of DNA bases. If repaired inefficiently, these DNA modifications result in deleterious mutations (Ichihashi et al. 2003). The mutagenic potential of ROS is enhanced further by oxidation of proteins and lipids, which can lead to altered signaling and cell proliferation (Bickers and Athar 2006).

Low levels of DNA damage initiate cell cycle arrest and removal of DNA lesions by nucleotide excision repair (Ichihashi et al. 2003). If DNA damage exceeds the repair

Corresponding authors.

${ }^{4}$ E-MAIL sabine.werner@cell.biol.ethz.ch; FAX 41-44-633-1174.

${ }^{5}$ E-MAIL matthias.schaefer@cell.biol.ethz.ch; FAX 41-44-633-1174.

Article is online at http://www.genesdev.org/cgi/doi/10.1101/gad.568810. capacity, apoptosis is initiated. In addition, apoptosis is induced by high levels of ROS and by death receptor signaling (Kulms et al. 2002). This prevents the persistence and spreading of DNA mutations and thus cancer formation, but, on the other hand, can lead to severe tissue damage.

As ROS contribute to UVB-induced photocarcinogenesis and apoptosis, it is of major importance to unravel the mechanisms of ROS detoxification. One of the most important players in the defense of cells against oxidative stress is the transcriptional regulator NF-E2-related factor 2 (Nrf2), which activates the expression of a battery of ROS-detoxifying enzymes and stimulates the production of antioxidants, including the tripeptide glutathione (GSH). GSH is one of the most versatile cellular antioxidants, and is produced in a two-step reaction from cysteine, glutamic acid, and glycine (Supplemental Fig. S1A). Interestingly, all enzymes involved in its biosynthesis are under the control of Nrf2 (Lu 2009). Due to its antioxidant properties, GSH is likely to be important for the prevention of UV-induced oxidative damage (Tyrrell and Pidoux 1988). This hypothesis is supported by the severe GSH depletion of keratinocytes in response to UVB 
irradiation (Zhu and Bowden 2004), which in turn enhances the susceptibility to UV-induced damage (Hanada et al. 1991).

We showed previously that Nrf2 is constitutively expressed in keratinocytes of normal skin and is upregulated during wound healing (Braun et al. 2002). Using Nrf2 knockout mice as well as mice expressing a dominantnegative Nrf2 mutant in the epidermis, we found that the constitutive, basal activity of Nrf2 in the skin is crucial for normal wound repair and for prevention of skin carcinogenesis (Braun et al. 2002; auf dem Keller et al. 2006). Nrf2 can be further activated by electrophiles, and possibly also by ROS (Beyer et al. 2007). In response to low doses of UVB or long-wave UVA irradiation of a mouse keratinocyte cancer cell line and of human fibroblasts and keratinocytes, increased Nrf2 activity was detected (Kannan and Jaiswal 2006; Gruber et al. 2010). Physiological doses of UVB, in contrast, did not activate Nrf2 in primary mouse keratinocytes or in murine back skin epidermis (Durchdewald et al. 2007). Recent results even indicated repression of Nrf2 activity by high UVB doses in normal human keratinocytes (Marrot et al. 2008; Kokot et al. 2009). The basal activity of Nrf2 seems to be required for protection of keratinocytes from UV cytotoxicity, since Nrf2 knockout mice showed a higher rate of apoptosis and oxidative damage in ear skin keratinocytes after UVB irradiation (Kawachi et al. 2008). Vice versa, topical treatment of SKH-1 mouse back skin with broccoli sprout extracts that contain the Nrf2 activator sulforaphane protected against UVB-induced photocarcinogenesis (Dinkova-Kostova et al. 2006). Since sulforaphane has multiple anti-carcinogenic effects independent of Nrf2 (Fimognari and Hrelia 2007; Dickinson et al. 2009), the effect of Nrf2 activation in the UV response needs to be studied using genetic approaches. This will also allow the identification of the molecular and cellular mechanisms underlying the protective effect of Nrf2 in the UVB response, which are as yet unknown. Activation of Nrf2 by ablation of its inhibitor kelch-like ECH-associated protein 1 (Keap1) causes postnatal lethality (Wakabayashi et al. 2003). Therefore, Keap1-deficient mice cannot be used to study the role of Nrf2 activation in UVB protection. Furthermore, Keap1 has additional binding partners besides Nrf2, and Nrf2-independent functions therefore cannot be excluded (Lee et al. 2009).

To circumvent this problem, we established a novel strategy to determine the consequences of Nrf2 target gene activation in keratinocytes in vivo by targeted expression of a constitutively active (ca) Nrf2 mutant. Our results show that Nrf2 activation is a potent strategy to protect the skin from UV damage and unravel a gradient of UVB protection in the skin that is controlled by Nrf2.

\section{Results}

Generation of transgenic mice expressing a caNrf2 mutant in keratinocytes

To specifically activate Nrf2 in the epidermis, we generated transgenic mice expressing a caNrf2 mutant in keratinocytes. A caNrf2 cDNA was cloned, which lacks the N-terminal Neh2 domain responsible for binding to the inhibitory protein Keap1 (Fig. 1A; Itoh et al. 1999). Transfection of COS-1 cells with a caNrf2 expression vector revealed nuclear localization of caNrf2 (Fig. 1B). Luciferase reporter assays were performed after cotransfection of HEK293T cells with a CMV-caNrf2 expression plasmid and a luciferase reporter plasmid containing the 51-base-pair (bp) antioxidant response element (ARE) of the rat $\mathrm{NAD}(\mathrm{P}) \mathrm{H}$ dehydrogenase quinone-1 (Nqo1) gene. AREs are cis-acting elements in the promoters of Nrf2 target genes. They are the binding sites for Nrf2, and mediate Nrf2-induced gene expression (Wasserman and Fahl 1997). Strong luciferase expression was found in cotransfected cells compared with cells transfected with the $A R E(N q O 1)$-Luc reporter alone (Fig. 1C).

The caNrf2 cDNA was cloned into an expression cassette with a $\beta$-actin promoter, a transcription/translation STOP cassette flanked by $\operatorname{lox} P$ sites, an internal ribosomal entry site (IRES), and the EGFP cDNA (Fig. 1D). Five transgenic founder mice (named $\beta$ ACTcaNrf2; FVB/N background) with different integration sites were generated by pronucleus injection. To induce caNrf2 expression in the epidermis and hair follicles, $\beta$ ACTcaNrf2 mice were mated with transgenic mice expressing Cre recombinase under control of the keratin 5 (K5) promoter (C57BL/6 background) (Ramirez et al. 2004). RNase protection assays (RPAs) using skin RNA showed that double-transgenic mice (named K5cre-caNrf2 mice) express the caNrf2 transgene at similar or lower levels compared with the endogenous Nrf2 gene (Fig. 1E). Proportional to the expression level of caNrf2, up-regulation of the established Nrf2 target genes Nqo1, Gsta3 (glutathione S-transferase 3), Gclc (glutamate cysteine ligase, catalytic subunit), and Srxn1 (sulfiredoxin1) (Kensler et al. 2007; Soriano et al. 2008) was detected by RPA or quantitative RT-PCR (qRT-PCR) (Fig. 1F,G). PCR amplification of the STOP cassette and adjacent nucleotides identified a 424-bp fragment in the epidermis of K5cre-caNrf2 transgenic mice, demonstrating excision of the STOP cassette (Fig. 1H). Since the STOP cassette is excised in the basal layer of the epidermis and hair follicles, caNrf2 is expressed in all layers in the course of epidermal self-renewal. This was verified by mating of K5cre-caNrf2 mice with AREhPAP reporter mice (Johnson et al. 2002). They carry a transgene with the ARE of the rat Nqo1 gene, an initiator element containing a minimal promoter and the human placental alkaline phosphatase (hPAP) cDNA. Strong hPAP activity was seen in all viable layers of the epidermis and hair follicles of triple-transgenic mice, demonstrating expression and activity of caNrf2 in keratinocytes in vivo (Fig. 1I).

\section{Mild hyperkeratosis in K5cre-caNrf2 transgenic mice}

K5cre-caNrf2 transgenic mice have normal growth and body weight. Between postnatal day 5 (P5) and P8, enhanced scaling was observed (Fig. 1J) that was no longer visible at later stages due to the dense fur. Histological analysis showed a similar thickness of the viable layers of the epidermis, but a slightly thicker cornified layer 
A

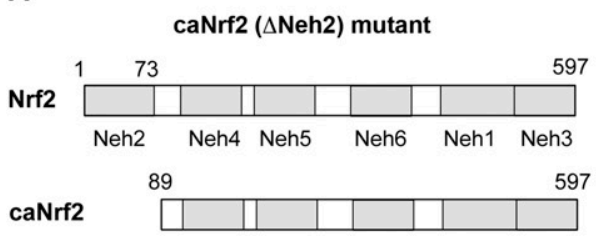

B

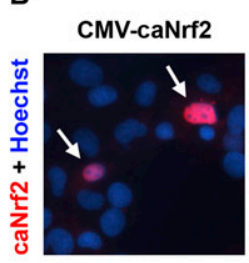

C

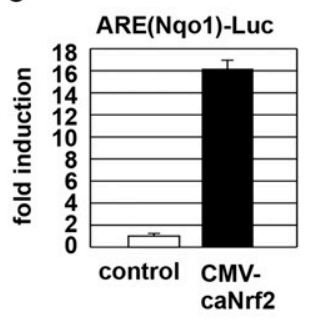

D

K5cre-caNrf2 mice

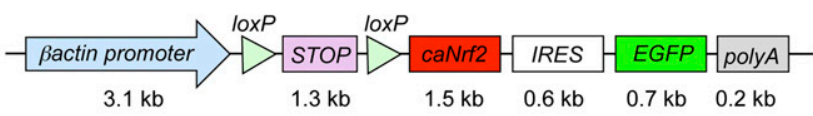

E

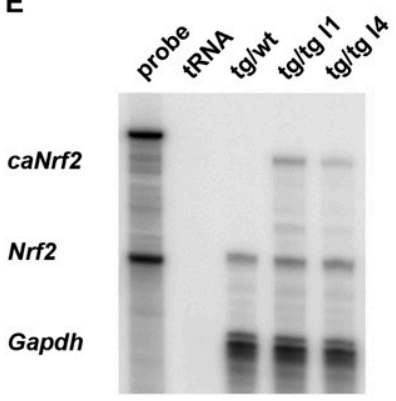

F

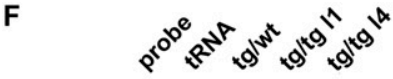

Nqo1

Gapdh

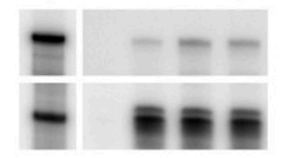

G

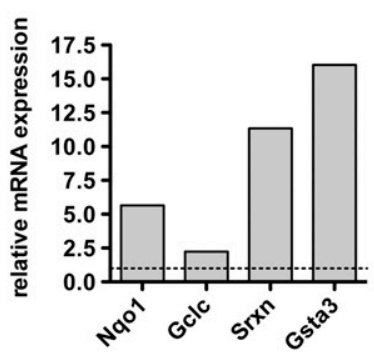

H

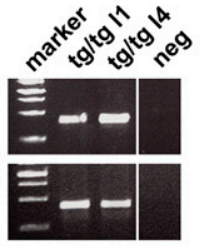

I

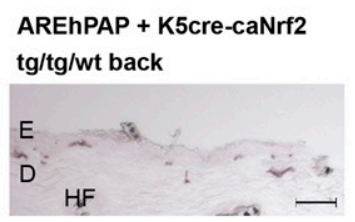

tg/tg/tg back

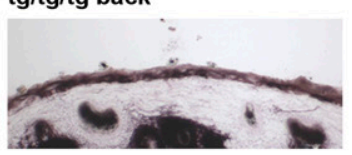

$\mathrm{tg} / \mathrm{tg} / \mathrm{tg}$ tail

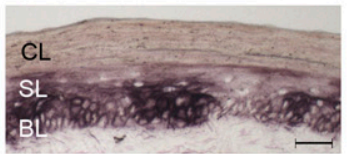

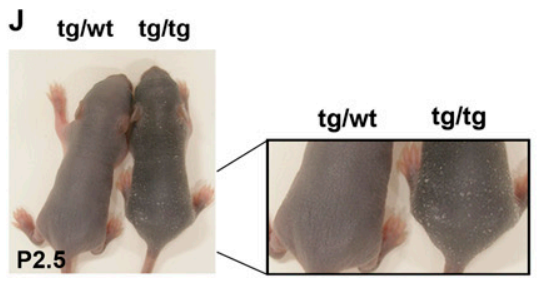
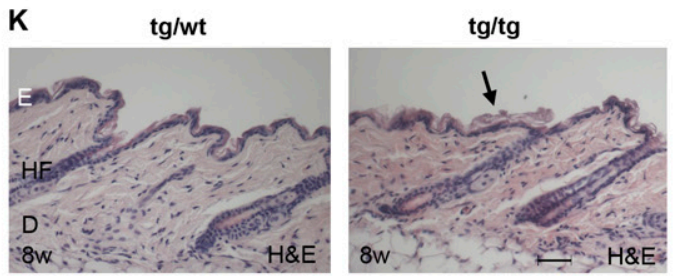

Figure 1. Generation of K5cre-caNrf2 transgenic mice. (A) Functional domains of Nrf2 and caNrf2 $(\Delta \mathrm{Neh} 2$ mutant). $(B)$ Immunofluorescence staining for Nrf2 in COS-1 cells transfected with a CMV-caNrf2 plasmid. Arrows point to nuclear caNrf2. (C) Luciferase reporter assay with a CMV-caNrf2 vector. The control is set to 1 . $(D)$ Scheme of the transgene used for generation of K5crecaNrf2 mice. $(E, F)$ RPA for caNrf2/Nrf2 $(E)$ and Nqo1 $(F)$ using RNAs from skin of transgenic/wild-type (tg/wt) and transgenic/ transgenic $(\mathrm{tg} / \mathrm{tg})$ mice of lines 1 and 4 . tRNA served as negative control (labeled "tRNA"); $10^{4}$ counts per minute (cpm) of the hybridization probes were used as marker (labeled "probe"). Note up-regulation of Nqo1 proportional to the caNrf2 expression level. (G) qRT-PCR of the Nrf2 target genes Nqo1, Gclc, Srxn1, and Gsta3 using epidermal RNAs of $\mathrm{tg} / \mathrm{tg}$ and tg/wild-type mice (set to 1). (H) PCR amplification of the STOP cassette ( $424 \mathrm{bp}$, top lane) and the Cre recombinase cDNA (419 bp, bottom lane) using DNA from mice of transgenic lines 1 and 4. (I) Staining for hPAP activity in back skin of double-transgenic AREhPAP/K5cre mice $(\mathrm{tg} / \mathrm{tg} / \mathrm{wt})(\mathrm{top}$ panel) and triple-transgenic AREhPAP/K5cre-caNrf2 mice ( $\mathrm{tg} / \mathrm{tg} / \mathrm{tg}$ ) (middle panel), and tail skin of triple-transgenic AREhPAP/K5cre-caNrf2 mice $(\mathrm{tg} / \mathrm{tg} / \mathrm{tg}$ ) (bottom panel). Bars: top panel, $50 \mu \mathrm{m}$; bottom panel, $30 \mu \mathrm{m}$. (J) P2.5 tg/wild-type and tg/tg K5cre-caNrf2 mice in overview (left picture) and close-up (right picture) of lower back. Note enhanced scaling in $\mathrm{tg} / \mathrm{tg}$ mice. (K) Longitudinal sections of back skin of 8 -wk-old $(8 \mathrm{w}) \mathrm{tg} /$ wild-type and $\mathrm{tg} / \mathrm{tg}$ mice. The arrow points to the area with the thicker cornified layer. Bar, $100 \mu \mathrm{m}$. (BL) Basal layer; (CL) cornified layer; (D) dermis; (E) epidermis; (HF) hair follicle; (SL) suprabasal layer. 
postnatally and throughout adulthood (Fig. 1K; data not shown). Morphometric analysis of $2-\mu \mathrm{m}$ paraffin sections of K5cre-caNrf2 mice confirmed the hyperkeratosis (Supplemental Fig. S1B). This phenotype is similar but less severe compared with Keap1 knockout mice (Wakabayashi et al. 2003), which further demonstrates the expected activity of the caNrf2 transgene in vivo. Keap1 knockout mice die postnatally from malnutrition due to severe hyperkeratosis in the upper digestive tract (Wakabayashi et al. 2003). K5cre-caNrf2 transgenic mice, in contrast, showed no hyperscaling in the esophagus and oral epithelium, due to low K5 promoter activity and consequent low caNrf2 expression levels in these epithelia (data not shown). Thus, mice expressing caNrf2 in keratinocytes are viable and can be used to investigate the consequences of enhanced Nrf2 activity in adult mice.

\section{caNrf2 protects keratinocytes from UVB cytotoxicity through enhanced ROS detoxification}

Primary keratinocytes from K5cre-caNrf2 mice showed enhanced Nrf2 activity, as verified by hPAP staining of keratinocytes from K5cre-caNrf2/AREhPAP triple-transgenic mice (Fig. 2A). Their rate of apoptosis was significantly lower compared with controls. This was already observed under normal culture conditions, reflecting the protection from stress that is imposed on culturing. Most importantly, caNrf2 protected keratinocytes from the toxicity of the ROS-producing agent menadione (Fig. 2B).

Upon irradiation of adult mice with a physiological dose of UVB $\left(100 \mathrm{~mJ} / \mathrm{cm}^{2}\right)$, we found a significant reduction in the number of apoptotic keratinocytes in the epidermis of K5cre-caNrf2 transgenic mice compared with control mice $24 \mathrm{~h}$ post-irradiation (hpi), as determined by staining for cleaved caspase-3 (Fig. 2C). To determine the mechanisms underlying this UV-protective effect, intracellular ROS levels were determined in UVB-irradiated cultured keratinocytes. For this purpose, cells were treated with $\mathrm{H}_{2}$ DCF-DA $\left(2^{\prime}, 7^{\prime}\right.$-dichlorodihydrofluorescein diacetate), and DCF fluorescence was analyzed by flow cytometry. ROS levels were significantly lower in immortalized (Fig. 2D) as well as primary keratinocytes (data not shown) from K5cre-caNrf2 transgenic mice compared with keratinocytes from control mice after irradiation with $10-40 \mathrm{~mJ} / \mathrm{cm}^{2} \mathrm{UVB}$. The effect of caNrf2 on direct UVB-induced DNA damage and its repair was analyzed by staining for cyclobutyl pyrimidine dimers (CPDs), which are the most predominant lesions induced by direct interference of UVB light with the DNA (Verschooten et al. 2006). Five minutes post-irradiation (mpi) and 24 hpi with $100 \mathrm{~mJ} / \mathrm{cm}^{2} \mathrm{UVB}$, no difference in the number of CPD-positive keratinocytes was found between control and K5cre-caNrf2 transgenic mice (Fig. $2 \mathrm{E})$. Immunohistochemical detection of p53, which is stabilized by DNA damage and ROS (Liu et al. 2008), showed a reduced number of positive cells in K5cre-caNrf2 mice (Fig. 2F). These findings strongly suggest that caNrf2 protects keratinocytes from UVB-induced apoptosis by reduction of intracellular ROS and subsequent ROSinduced DNA damage, but has no influence on formation and repair of lesions resulting from direct DNA modification by UVB.

A gradient of $U V B$-induced apoptosis in the murine epidermis: correlation with Nrf2 expression and activity

Histological analysis of hematoxylin/eosin (H\&E)-stained $2-\mu \mathrm{m}$ paraffin sections from UVB-irradiated wild-type mouse back skin showed that $85 \%$ of (apoptotic) sunburn cells are located in the basal layer, and only $15 \%$ are located in the suprabasal layers $(N=11)$ (Fig. 3A). Sunburn cells are apoptotic keratinocytes, which can be identified by their pyknotic nuclei and the eosinophilic cytoplasm (Young 1987). In the much thicker tail skin epidermis, $63 \%$ of the sunburn cells were in the basal layer, $26 \%$ were in the lower suprabasal layers, and $11 \%$ were in the upper suprabasal layers $(N=6)$ (Fig. 3B). This was confirmed by staining for cleaved caspase-3 $(58 \%$ basal layer, $37 \%$ lower suprabasal layers, $5 \%$ upper suprabasal layers, $N=6$ ) (Fig. 3C; data not shown) and p53 (data not shown). Thus, apoptotic keratinocytes are distributed in a basal-to-suprabasal gradient (see below; Fig. 3J).

To determine if the reduced apoptosis of suprabasal keratinocytes results from enhanced Nrf2 activity and subsequent ROS detoxification, we compared the expression levels of Nrf2 and established Nrf2 target genes between suprabasal and basal keratinocytes. Suprabasal cell layers of tail skin were separated from the basal layer by chymotrypsin treatment, as described for human skin (Sasai et al. 1979). Successful separation was verified histologically, by K10 immunostaining (Fig. 3D), and by qRT-PCR for the late differentiation marker Sprr2a (Fig. 3E; Song et al. 1999). In the samples obtained from the first separation $(19 \times$ Sprr2a suprabasal vs. basal keratinocytes), qRT-PCR showed a much higher expression of Nrf2 and its target genes Nqo1, Gclc, Gclm (glutamate cysteine ligase, modifier subunit), Gsr (glutathione reductase), and Srxn1 in suprabasal compared with basal keratinocytes (Fig. 3E). Gss (glutathione synthetase) was expressed at similar levels in basal and suprabasal keratinocytes, while Gsta3 had a lower expression level in suprabasal cells. In a more efficient separation $(588 \times$ Sprr2a suprabasal vs. basal keratinocytes), an even higher enrichment of Nrf2, Nqo1, Gclc, Gclm, Gsr, and Srxn1 was observed in suprabasal keratinocytes. In this experiment, Gss and Gsta3 showed equal expression levels in suprabasal and basal keratinocytes (Fig. 3E). For Nqo1, a suprabasal-to-basal gradient was confirmed on the protein level by immunofluorescence staining (Fig. 3F).

Studies with Nrf2 knockout mice revealed that the differential expression of Nqo1, Gclc, Gclm, and Gsr is indeed Nrf2-dependent, since expression levels of these genes were similar in basal and suprabasal keratinocytes in the absence of Nrf2 (Fig. 3G). Only Srxn1 showed a higher expression in suprabasal compared with basal cells of Nrf2 knockout animals. Most importantly, the steep gradient in the distribution of sunburn cells that is seen in wild-type mice 24 hpi with UVB was much less pronounced in Nrf2 knockout mice. This was due predominantly to a strong 
A

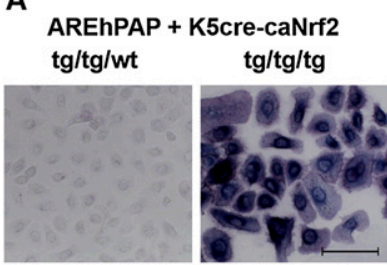

B

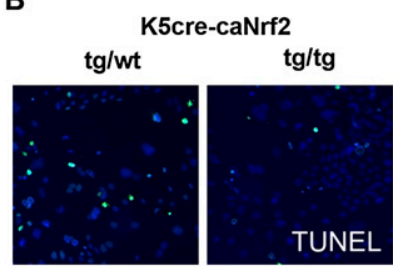

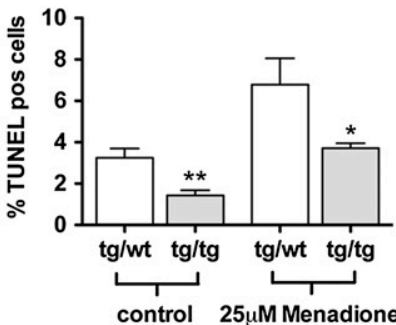
control

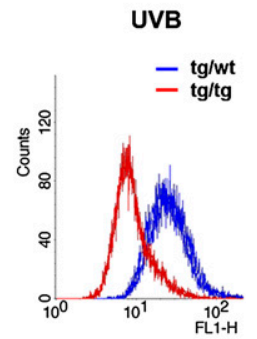

E
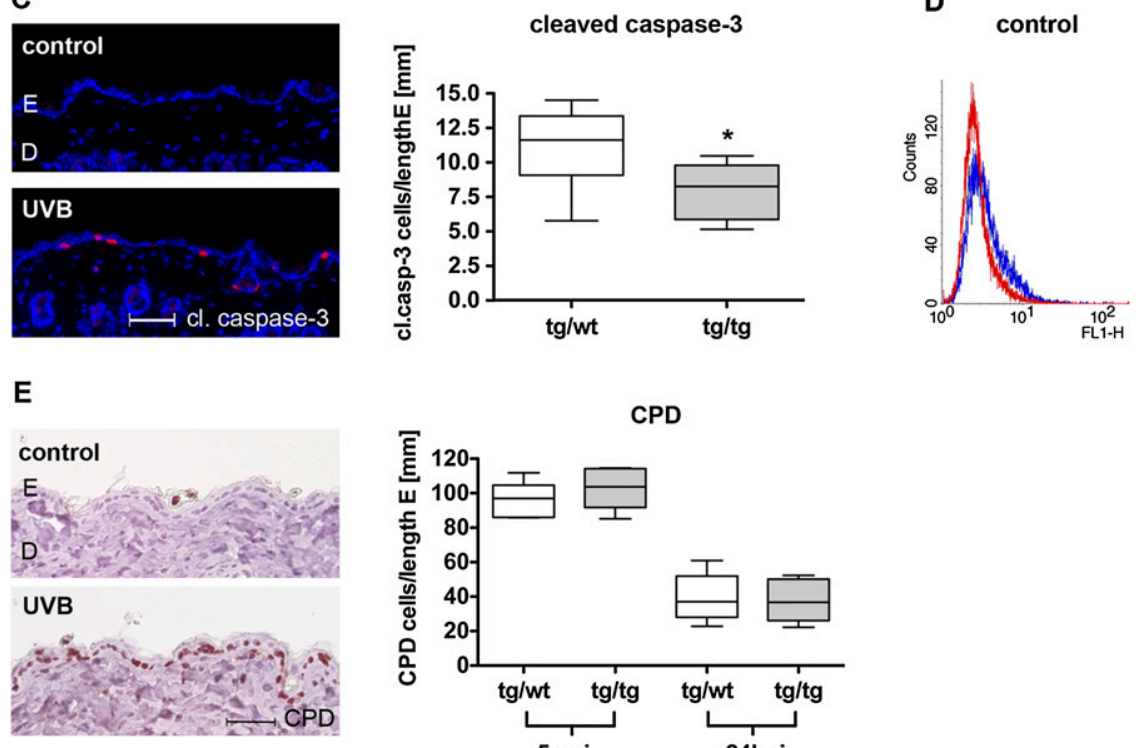

CPD
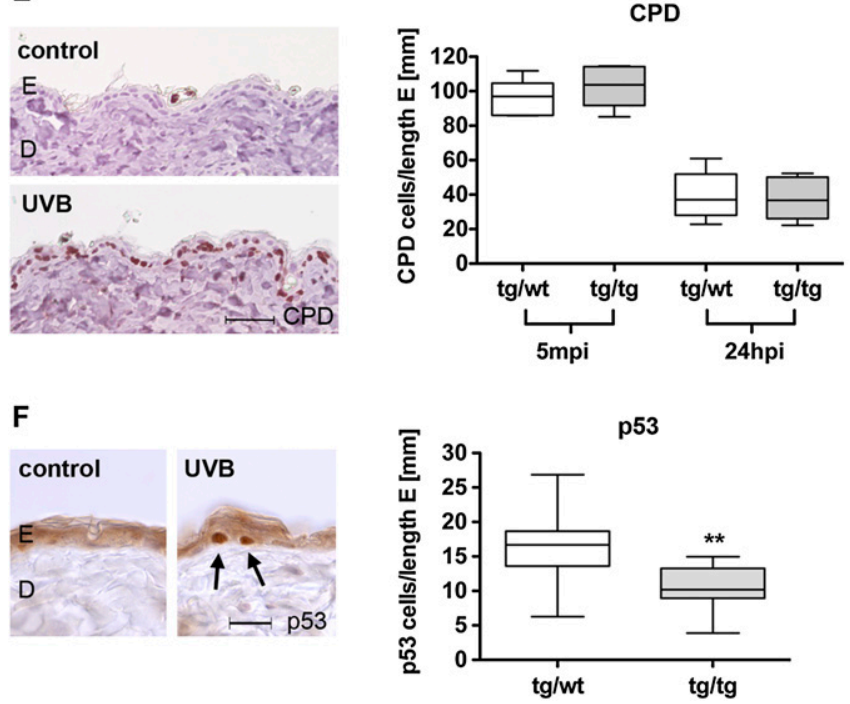

Figure 2. ROS detoxification and protection from UVB-induced apoptosis in K5cre-caNrf2 transgenic mice. $(A)$ hPAP activity in primary keratinocytes of double-transgenic AREhPAP/K5cre mice $(\mathrm{tg} / \mathrm{tg} / \mathrm{wt})$ and triple-transgenic AREhPAP/K5cre-caNrf2 mice $/ \mathrm{tg} / \mathrm{tg} /$ $\mathrm{tg})$. Bar, $100 \mu \mathrm{m}$. (B, left panel) TUNEL staining of primary keratinocytes (green), nuclei of tg/wild-type mice (left), and tg/tg K5crecaNrf2 mice (right) stained with Hoechst (blue). (Right graph) Percentage of TUNEL-positive keratinocytes of tg/wild-type and tg/tg mice without (control) $(P=0.0012)$ and after $8 \mathrm{~h}$ of treatment with $25 \mu \mathrm{M}$ menadione $(P=0.0350)$. ( $C$, left) Immunofluorescence for cleaved caspase-3 (red), counterstained with Hoechst (blue), in nonirradiated (control) skin and in UVB-irradiated back skin (UVB) (24 hpi, $100 \mathrm{~mJ} / \mathrm{cm}^{2}$ ) of wild-type mice. Bar, $50 \mu \mathrm{m}$. (Right) Number of cleaved caspase-3-positive cells per length epidermis in tg/wild-type and $\mathrm{tg} / \mathrm{tg}$ mice, $24 \mathrm{hpi}, 100 \mathrm{~mJ} / \mathrm{cm}^{2} \mathrm{UVB}(N=9, P=0.019)$. (D) FACS analysis of DCF in control (nonirradiated) and $20 \mathrm{~mJ} / \mathrm{cm}^{2} \mathrm{UVB}-$ irradiated immortalized keratinocytes of tg/wild-type (blue) and $\mathrm{tg} / \mathrm{tg}$ (red) K5cre-caNrf2 mice. (E, left) Staining for CPD-positive cells in the epidermis of nonirradiated (control) and UVB-irradiated (UVB) wild-type mice. Bar, $50 \mu \mathrm{m}$. (Right) CPD-positive cells per length epidermis, $5 \mathrm{~min}$ (left side) and $24 \mathrm{hpi}$ (right side) with $100 \mathrm{~mJ} / \mathrm{cm}^{2} \mathrm{UVB}$ in tg/wild-type and tg/tg K5cre-caNrf2 transgenic mice $(N=6)$. $(F$, left) Staining for p53-positive cells in the epidermis of nonirradiated (control) and UVB-irradiated (UVB) wild-type mice. Bar, $25 \mu \mathrm{m}$. (Right) p53-positive cells per length epidermis in tg/wild-type and $\mathrm{tg} / \mathrm{tg}$ K5 cre-caNrf2 transgenic mice, $24 \mathrm{hpi}, 100 \mathrm{~mJ} / \mathrm{cm}^{2} \mathrm{UVB}(\mathrm{N}=11$, $P=0.0039$ ).

increase in the number of apoptotic keratinocytes in the suprabasal layers of the knockout mice (Fig. $3 \mathrm{H}$ ). This finding is consistent with the decrease in the expression of Nrf2 target genes in suprabasal relative to basal keratinocytes (see Fig. 3G). Surprisingly, a minor decrease in the number of apoptotic basal cells was observed in Nrf2 knockout mice compared with wild-type controls (Fig. $3 \mathrm{H})$, possibly due to activation of compensatory mechanisms in basal cells that may involve other Nrf family members (Braun et al. 2002). 

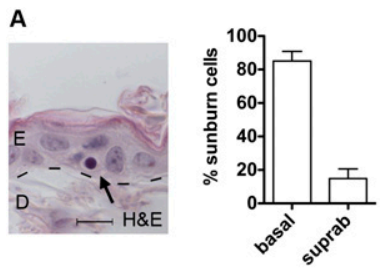

B
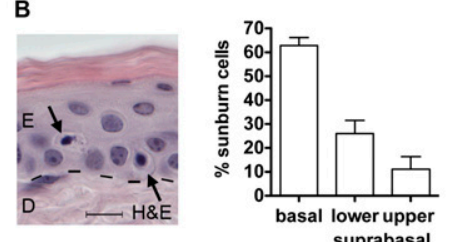

C

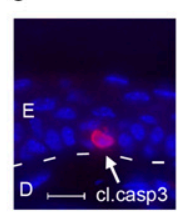

E

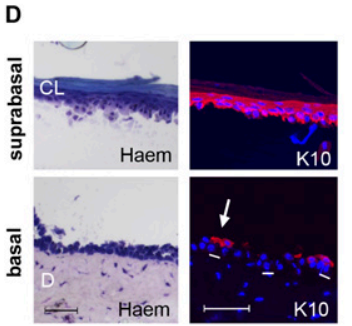

$\mathbf{F}$
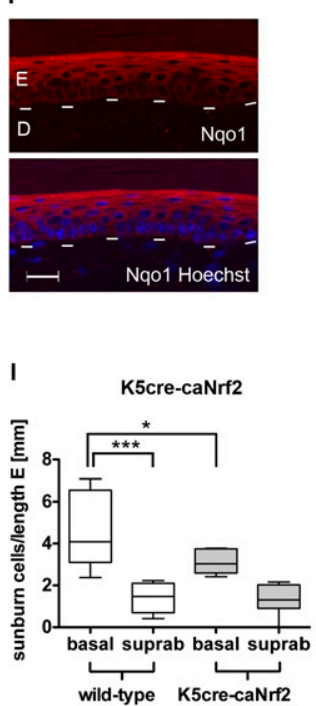

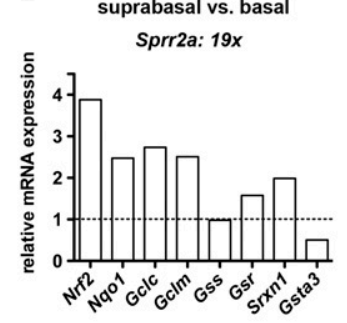

G

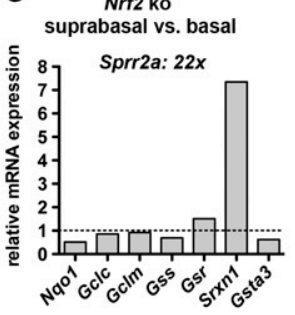

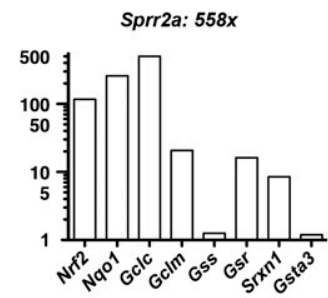

H

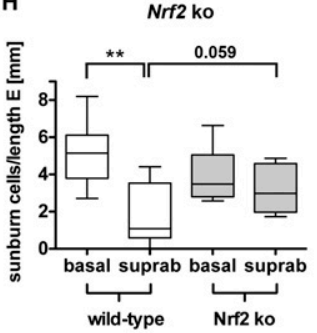

J
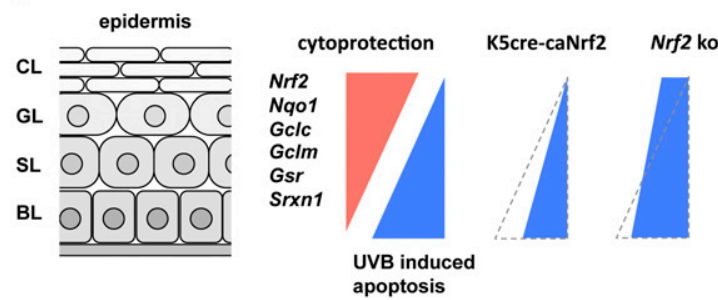

Figure 3. UVB-induced apoptosis and Nrf2-mediated cytoprotection in the murine epidermis. $(A, B, l e f t) \mathrm{H} \& \mathrm{E}$ staining of wild-type mouse back skin $(A)$ and tail skin (B), $24 \mathrm{hpi}, 100 \mathrm{~mJ} / \mathrm{cm}^{2}$ UVB. Arrows point to sunburn cells in the basal $(A, B)$ and lower suprabasal $(B)$ layers. Bars: $A, B, 10 \mu \mathrm{m}$. (Right side) Distribution of sunburn cells in back skin $(A)(N=11)$ and tail skin $(B)$ $(N=6)$ epidermis in percent. $(C)$ Cleaved caspase-3 staining of wild-type mouse tail skin, $24 \mathrm{hpi}, 100 \mathrm{~mJ} / \mathrm{cm}^{2} \mathrm{UVB}$. The arrow points to a positive keratinocyte. Bar, $15 \mu \mathrm{m}$. (D) Tail skin after chymotrypsin treatment stained with rapid differential hematology staining (Haem, left panel) or immunostained for K10 (red), costained with Hoechst (blue) (right panel). (Top picture) Suprabasal layers. (Bottom picture) Basal layer and dermis. The arrow points to remaining K10-positive suprabasal keratinocytes. Bars, $50 \mu \mathrm{m}$. (E) Nrf2, Nqo1, Gclc, Gclm, Gss, Gsr, Srxn1, and Gsta3 expression in suprabasal relative to basal keratinocytes of tail epidermis from wildtype mice shown for two different separations, analyzed by qRT-PCR. Expression levels in basal keratinocytes were set to 1 , indicated by dashed line. $(F)$ Immunofluorescence analysis of $\mathrm{Nqo} 1$ (red) in tail skin, costained with Hoechst (blue). Note the staining gradient from basal to suprabasal. Bar, $25 \mu \mathrm{m}$. (G) Nqo1, Gclc, Gclm, Gss, Gsr, Srxn1, and Gsta3 expression in suprabasal relative to basal keratinocytes of tail epidermis from Nrf2 knockout (ko) mice, analyzed by qRT-PCR. Note reduced expression of NqO1, Gclc, Gclm, and Gsr, but elevated expression of Srxn1 in Nrf2 knockout compared with wild-type mice lleft graph in $E)$. $(H, I)$ Distribution of sunburn cells per length epidermis in Nrf2 knockout mice $(H)(N=8 / 9)$ and K5cre-caNrf2 mice $(I)(N=8)$ in back skin epidermis, 24 hpi with $100 \mathrm{~mJ} / \mathrm{cm}^{2}$ UVB. Note the significant difference between basal and suprabasal keratinocytes in wild-type mice $(P=0.0012$ for $H ; P=0.0002$ for $I)$, the strong increase in suprabasal sunburn cells $(P=0.059)$ but the slight decrease in basal sunburn cells in Nrf2 knockout mice $(H)$, and the strong decrease in basal sunburn cells in K5cre-caNrf2 mice $(I)(P=0.0499)$. ( $/$ ) Scheme demonstrating the gradient of ROS detoxification and UVB-induced apoptosis in wild-type and Nrf2 mutant mice. In wild-type mice, suprabasal keratinocytes express higher levels of Nrf2 and its cytoprotective target genes compared with basal keratinocytes. This results in preferential UVB-induced apoptosis of basal cells. In K5cre-caNrf2 transgenic mice, basal cells are protected from apoptosis, resulting in reduced numbers of mainly basal apoptotic cells and reduction of the apoptotic gradient. In Nrf2 knockout mice, the gradient is also abolished, mainly due to strongly enhanced apoptosis of suprabasal cells but slightly reduced apoptosis of basal cells. (BL) Basal layer; (SL) spinous layer; (GL) granular layer; (CL) cornified layer.

These findings revealed that endogenous Nrf2 establishes a suprabasal-to-basal expression gradient of many ROS-detoxifying genes, which controls at least in part the basal-to-suprabasal gradient of UVB-induced apoptosis (shown schematically in Fig. 3J).

In support of this hypothesis, pan-epidermal activation of Nrf2 in K5cre-caNrf2 transgenic mice, which results in similar activity of Nrf2 in all epidermal layers, also reduced the gradient of apoptosis due to a preferential reduction in the number of apoptotic cells in the basal layer (Fig. 3I,J).

\section{Generation of mice expressing caNrf2 in suprabasal keratinocytes}

To enforce the basal-to-suprabasal gradient of cytoprotection in the epidermis, we next generated transgenic mice expressing caNrf2 only in suprabasal keratinocytes using the bovine keratin $10(\mathrm{~K} 10)$ promoter and a previously described expression cassette (Fig. 4A; Werner et al. 1993). Three transgenic founder mice were generated with lower or similar expression of caNrf2 compared with endogenous Nrf2 (Supplemental Fig. S1C). They showed 
A

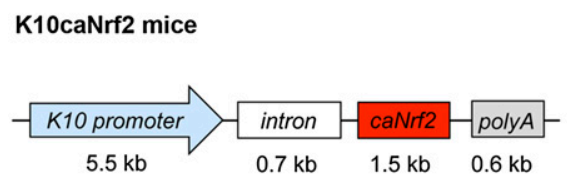

B

AREhPAP + K10caNrf2

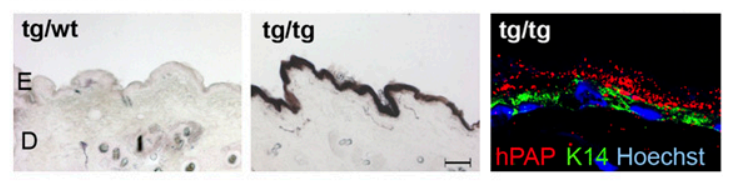

E
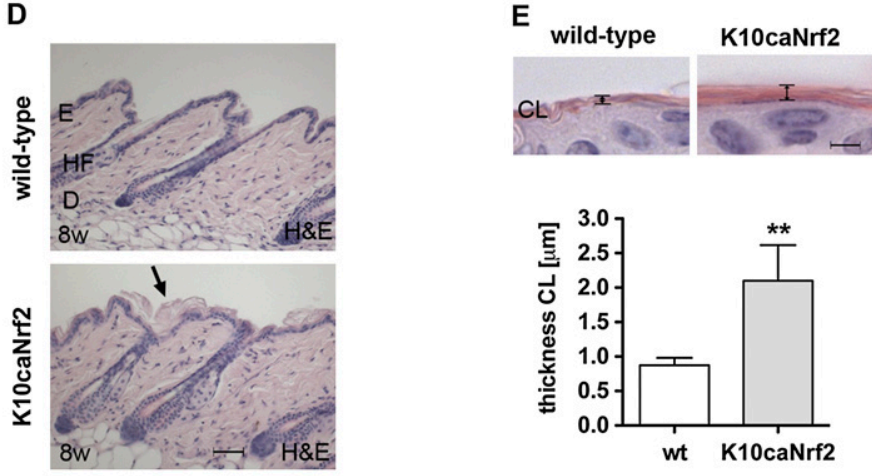

G

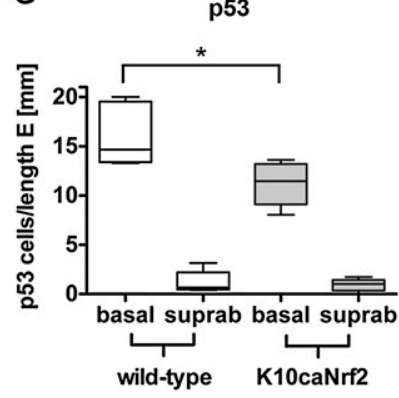

H

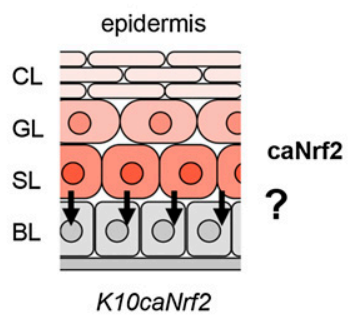

Figure 4. Generation of K10caNrf2 transgenic mice and protection from UVB-induced apoptosis. $(A)$ Scheme of the transgene used for generation of transgenic mice. $(B)$ NBT/BCIP staining for hPAP activity in back skin of AREhPAP transgenic mice (left panel) and AREhPAP/K10caNrf2 double-transgenic mice (middle panel). Bar, $50 \mu \mathrm{m}$. (Right panel) Fluorescence staining for hPAP activity (red), immunofluorescence for K14 (green), and counterstaining with Hoechst (blue) on back skin sections of AREhPAP/K10caNrf2 mice. Note nonoverlapping localization of hPAP staining in suprabasal and K14 staining in basal keratinocytes. $(C)$ Wild-type (wt) and K10caNrf2 transgenic mice at P2.5 in overview (top picture) and close-up (bottom picture) of lower back. Note enhanced scaling in K10caNrf2 mice. $(D)$ Longitudinal back skin sections of wild-type (wt) and K10caNrf2 (tg) mice at 8 wk (8w). The arrow points to the area with the thicker cornified layer. Bar, $100 \mu \mathrm{m}$. (E, top panel) H\&E staining of 2- $\mu \mathrm{m}$ sections of skin from wild-type and K10caNrf2 mice. Bar, $2 \mu \mathrm{m}$. (Graph) Morphometric measurement of the thickness of the cornified layer $(N=5, P=0.0025)$. $(F)$ Number of cleaved caspase-3-positive cells per length epidermis in 8- to 10 -wk wild-type and K10caNrf2 mice, $24 \mathrm{hpi}, 100 \mathrm{~mJ} / \mathrm{cm}^{2} \mathrm{UVB}(N=8, P=0.003)$. $(G)$ Position of p53-positive keratinocytes per length epidermis in 8 - to 10 -wk wild-type $(1$ feft $)$ and K10caNrf2 mice $(r i g h t)(N=5, P=$ 0.0317). (H) Scheme of caNrf2 expression in the epidermis of K10caNrf2 mice and proposed paracrine cytoprotection of basal keratinocytes by suprabasal keratinocytes, indicated by arrows.

strong up-regulation of the Nrf2 target genes Nqo1, Gclc, and Gst $\pi$ (Supplemental Fig. S1D). Mating of K10caNrf2 mice with AREhPAP mice showed a strong hPAP activity in the epidermis of double-transgenic mice (Fig. 4B). Fluorescence analysis of hPAP activity and K14 immunofluorescence showed selective hPAP expression in suprabasal keratinocytes (Fig. 4B).

Similar to K5cre-caNrf2 mice, K10caNrf2 transgenic mice showed mild hyperkeratosis at P2.5 (Fig. 4C), and a slightly thickened cornified layer (Fig. 4D). This was confirmed by morphometric analysis of $2-\mu \mathrm{m}$ paraffin sections of K10caNrf2 mice ( $N=5 / 7, P=0.0025$, Fig. 4E). The extent of hyperkeratosis was dependent on the genetic background: It was hardly detectable in K10caNrf2 trans- genic mice in a pure B6D2F1 background, but was very pronounced in a B6D2F1, C57BL/6J mixed background (Supplemental Fig. S1E). In spite of the hyperkeratosis, keratinocyte differentiation was not obviously altered, as determined by normal expression of K14, K10, K6, and loricrin (Supplemental Fig. S1F,G). This is consistent with the normal thickness of the viable part of the epidermis.

\section{Enhanced Nrf2 activity in suprabasal keratinocytes protects basal cells from UVB cytotoxicity}

When K10caNrf2 transgenic mice were irradiated with $100 \mathrm{~mJ} / \mathrm{cm}^{2} \mathrm{UVB}$, the number of apoptotic keratinocytes was significantly lower 24 hpi compared with wild-type 
mice, as determined by cleaved caspase-3 staining (Fig. 4F) and TUNEL assay (Supplemental Fig. S2A). The number of p53-positive keratinocytes was also significantly lower (Supplemental Fig. S2B). The anti-apoptotic effect was dose-dependent: While $20 \mathrm{~mJ} / \mathrm{cm}^{2}$ UVB caused a $60 \%$ reduction in the number of apoptotic cells in K10caNrf2 transgenic compared with wild-type mice, $100 \mathrm{~mJ} / \mathrm{cm}^{2}$ UVB led to only $28 \%$ reduction (Supplemental Fig. S2A). Surprisingly, the decrease in apoptosis was similar in K10caNrf2 and K5cre-caNrf2 transgenic mice (see Fig. 2C), in spite of the exclusive expression of caNrf2 in suprabasal keratinocytes of K10caNrf2 mice. Remarkably, a significant UVB protection-as shown by counting of p53-positive cells-was observed in the basal layer, where caNrf2 is not expressed (Fig. 4G; data not shown). This was not due to UVB-induced premature differentiation (Jiang et al. 2007) and consequent caNrf2 expression in the basal layer, since hPAP activity was still restricted to suprabasal keratinocytes after UVB irradiation of K10caNrf2/AREhPAP double-transgenic mice (Supplemental Fig. S2C). Moreover, it is unlikely to result from enhanced absorption of UVB by the thicker cornified layer in K10caNrf2 mice, since the number of apoptotic cells was similar after UVB irradiation of K10caNrf2 mice in the B6D2F1 background and the B6D2F1, C57BL/6 mixed background (Supplemental Fig. S2D), which show a different severity of hyperkeratosis (see Supplemental Fig. S1E). Finally, no difference in the inflammatory response was detected, as the number of neutrophils and mast cells and the expression of the proinflammatory cytokine $I L-1 \beta$ were not changed (Supplemental Fig. S2E,F; data not shown).

\section{caNrf2-mediated protection of neighboring}

keratinocytes is dependent on GSH

Based on these findings, we hypothesized that the protection of basal cells from UVB-induced apoptosis in K10caNrf2 mice is mediated by a paracrine-acting factor (Fig. $4 \mathrm{H}$ ), which is activated by Nrf2 in suprabasal keratinocytes. A possible candidate is GSH. To functionally test this hypothesis, K10caNrf2 transgenic mice were mated with mice lacking Gclm, the modifier subunit of the glutamate cysteine ligase (Yang et al. 2002). These mice can still produce GSH, since they still express the Gclc. However, Gclm knockout mice (C57BL/6 background) have an $84 \%-91 \%$ reduction in the levels of GSH in a variety of tissues (Yang et al. 2002). Gclmdeficient mice showed only a minor increase in the number of apoptotic cells after UVB irradiation compared with wild-type mice, as determined by staining for cleaved caspase-3 (Fig. 5A) and by TUNEL staining (Supplemental Fig. S3A). Apparently, the residual level of GSH is sufficient to protect keratinocytes from UVBinduced apoptosis. In K10caNrf2/Gclm double-mutant mice, however, the number of apoptotic cells was not reduced significantly compared with wild-type/Gclm knockout mice (Fig. 5A; Supplemental Fig. S3A). Thus the protective effect of caNrf2 was abolished in a Gclmdeficient mouse background.
Nrf2 controls a GSH biosynthesis/recycling pathway in the murine epidermis

One possible mechanism for how basal keratinocytes are protected by suprabasal keratinocytes expressing caNrf2 is secretion and import of the antioxidant GSH. So far there is little evidence for a direct cellular import of GSH. Therefore, cells need to import substrates for intracellular GSH synthesis-in particular, the rate-limiting amino acid cysteine (Dalton et al. 2004).

One mechanism to provide extracellular cysteine involves the formation of $\gamma$-glutamyl cystine conjugates and the dipeptide cysteine-glycine from GSH by the ectoenzyme $\gamma$-glutamyltransferase $(\gamma \mathrm{GT})$ (Supplemental Fig. S3B; Kinlough et al. 2005). However, $\gamma \mathrm{GT}$ activity was not detectable in the interfollicular epidermis of wild-type and K10caNrf2 transgenic mice before and after UVB irradiation using the chromogenic substrate $\gamma$-glutamyl-4-methoxy-2-naphtylamide (GMNA) (Fig. 5B; Rutenburg et al. 1969; De Young et al. 1978). Hair follicles, which are known to have active $\gamma \mathrm{GT}$ in the anagen phase, served as internal positive control /Chiba and Jimbow 1986).

Another mechanism to provide extracellular cysteine involves the glutamate/cystine antiporter $\mathrm{x}_{\mathrm{c}}{ }^{-}$, which mediates the uptake of cystine. The latter is intracellularly reduced to cysteine, which can be secreted to the extracellular space (see Fig. 6; Lo et al. 2008). qRT-PCR showed that K10caNrf2 transgenic mice express higher levels of the light subunit $x C T$ of the $\mathrm{x}_{\mathrm{c}}{ }^{-}$antiporter compared with wild-type mice (Fig. 5C). Furthermore, a higher expression of this gene was seen in suprabasal compared with basal keratinocytes of wild-type mice (see below; Fig. 5I), consistent with their stronger expression of Nrf2. This most likely leads to increased secretion of cysteine by suprabasal cells in wild-type mice, and particularly in K10caNrf2 transgenic mice (see Fig. 6). Extracellular cysteine is taken up by the ubiquitous alanine-serine-cysteine (Asc) transporter (Christensen 1990). Indeed, expression of Asct1 (Slc1a4) was also increased in the epidermis of $\mathrm{K} 10 \mathrm{caNrf} 2$ transgenic mice (Fig. 5C). Interestingly, this gene is expressed at higher levels in basal compared with suprabasal layers of wildtype mice (see below; Fig. 5I), consistent with the requirement of these cells to import cysteine.

Due to its high reactivity, extracellular cysteine is oxidized rapidly to cystine, which cannot be imported by Asc transporters (Toohey 1975). Extracellular cystine, however, can undergo a thiol/disulfide exchange reaction with GSH, resulting in formation of a GSH-cysteine conjugate and free cysteine (see Fig. 6; Wang and Cynader 2000). We investigated whether Nrf2 enhances the levels of extracellular GSH by GSH synthesis, recycling, and export from suprabasal keratinocytes. qRT-PCR revealed a strong increase in the mRNA levels of both $G c 1$ subunits (Gclc and Gclm), as well as of Gss and Gsr, in nonirradiated K10caNrf2 transgenic mice and 24 hpi with $100 \mathrm{~mJ} / \mathrm{cm}^{2}$ UVB (Fig. 5D; data not shown). Although the caNrf2positive suprabasal cells comprise only a minor percentage of the epidermal cells, GSH levels were reproducibly 
A

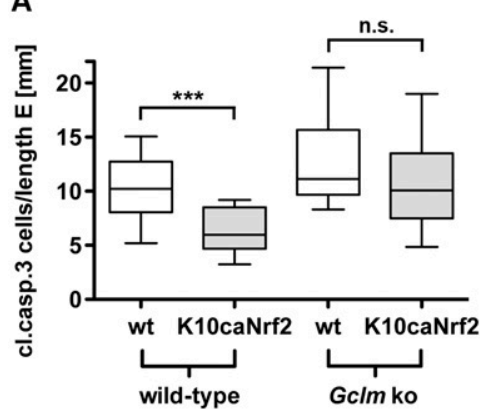

D

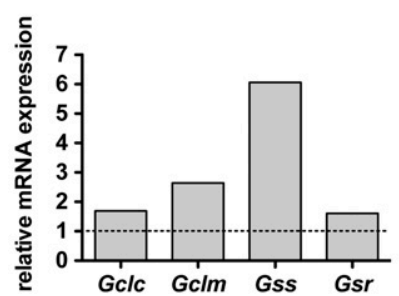

G

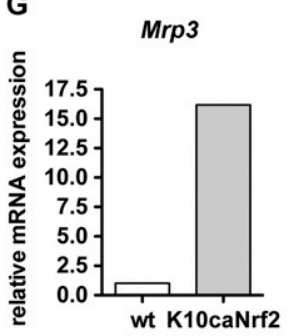

B

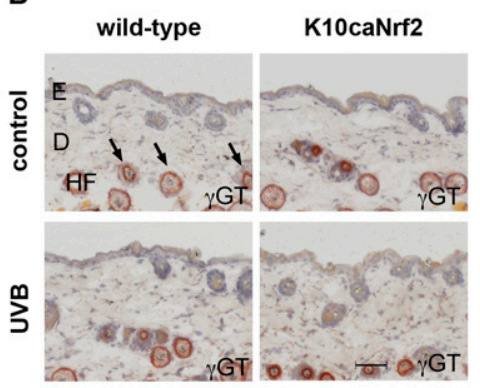

C

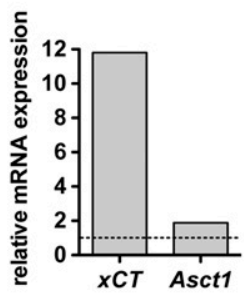

E

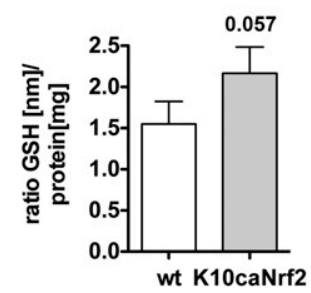

$\mathbf{F}$

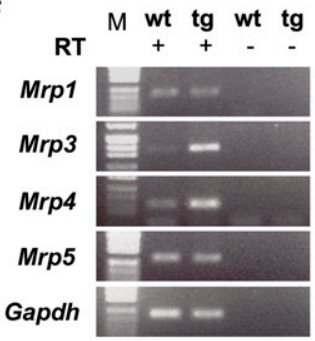

H

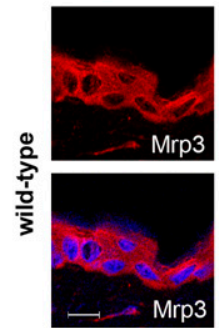

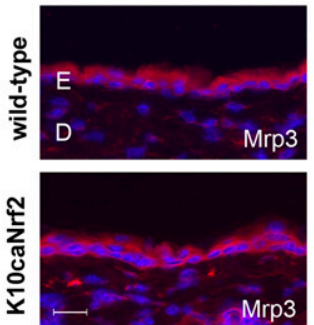

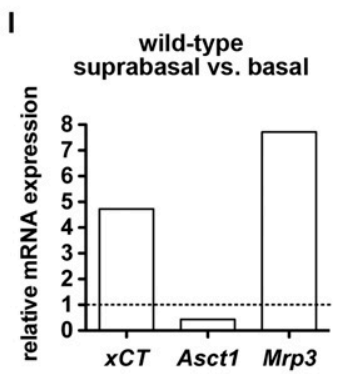

Figure 5. GSH metabolism in K10caNrf2 transgenic mice. (A) Cleaved caspase-3-positive cells per length epidermis, 24 hpi, $100 \mathrm{~mJ} /$ $\mathrm{cm}^{2}$ in wild-type and K10caNrf2 transgenic mice crossed with wild-type (left) $(N=15, P=0.0003)$ and Gclm knockout (right) mice $(N=$ $14, P=0.23) .(B) \gamma \mathrm{GT}$ activity staining (auburn) in back skin of wild-type and tg mice, nonirradiated (control) and $24 \mathrm{hpi}, 100 \mathrm{~mJ} / \mathrm{cm}^{2}$ UVB-irradiated (UVB) mice. Arrows point to staining in lower hair follicle (HF). No staining is visible in the interfollicular epidermis. Bar, $50 \mu \mathrm{m} .(C)$ qRT-PCR of $x C T$ and Asct1 in epidermis of tg versus wild-type mice (set to 1, indicated by dashed line). (D) qRT-PCR analysis of Gclc, Gclm, Gss, and Gsr using RNAs from epidermis of wild-type and tg mice. The expression level in wild-type mice was set to 1 , indicated by dashed line. The qRT-PCR data shown in $C$ and $D$ were obtained from pooled epidermal samples, and were reproduced with a different set of RNAs from different pools of mice. $(E)$ GSH levels normalized to total protein in keratinocytes of wild-type and tg mice $(N=5, P=0.0571)$. (F) RT-PCR of Mrp1, Mrp3, Mrp4, Mrp5 in wild-type epidermis with (+) and without (-) RT. $(G)$ qRT-PCR of $M r p 3$ in wild-type and tg mice. $(H)$ Immunofluorescence analysis of Mrp3 (red), counterstained with Hoechst (blue) in wild-type and tg mouse back skin. Bars: left panel, $15 \mu \mathrm{m}$; right panel, $30 \mu \mathrm{m}$. (I) xCT, Asct1, and Mrp3 expression in suprabasal relative to basal keratinocytes of wild-type mouse tail epidermis (average of two different separations), analyzed by qRT-PCR. (n.s.) Not significant.

higher in the epidermis of K10caNrf2 transgenic mice compared with wild-type mice (Fig. 5E). Consistent with previous observations (Li et al. 2006), semiquantitative RT-PCR revealed expression of the GSH exporters Mrp1, Mrp3, Mrp4, and Mrp5 in the murine epidermis (Fig. 5F). Mrp3 and Mrp4 were even up-regulated in K10caNrf2 transgenic mice (Fig. 5F,G; data not shown). Immunofluorescence analysis localized Mrp3 in the plasma membrane and cytoplasm of keratinocytes of wild-type and K10caNrf2 transgenic mice (Fig. 5H). In wild-type mice, it was expressed at much higher levels by suprabasal cells compared with basal cells (Fig. 5I), consistent with the enhanced expression of Nrf2 and in accordance with its postulated role in the export of GSH by suprabasal cells. In contrast, Mrp4 was detected only in the basal membrane of basal keratinocytes and therefore cannot be responsible for the export of GSH from suprabasal keratinocytes (data not shown).

These findings strongly suggest that suprabasal elevation of Nrf2 activity enhances production and secretion of GSH, possibly via Mrp3. In addition, cystine uptake and subsequent release of cysteine is increased. Together, this leads to increased availability of extracellular cysteine and its uptake by basal cells. This allows the production of GSH by these cells, which protects them from UVBinduced apoptosis. This pathway, as well as the role of Nrf2 and its regulation, are shown schematically in Figure 6. 


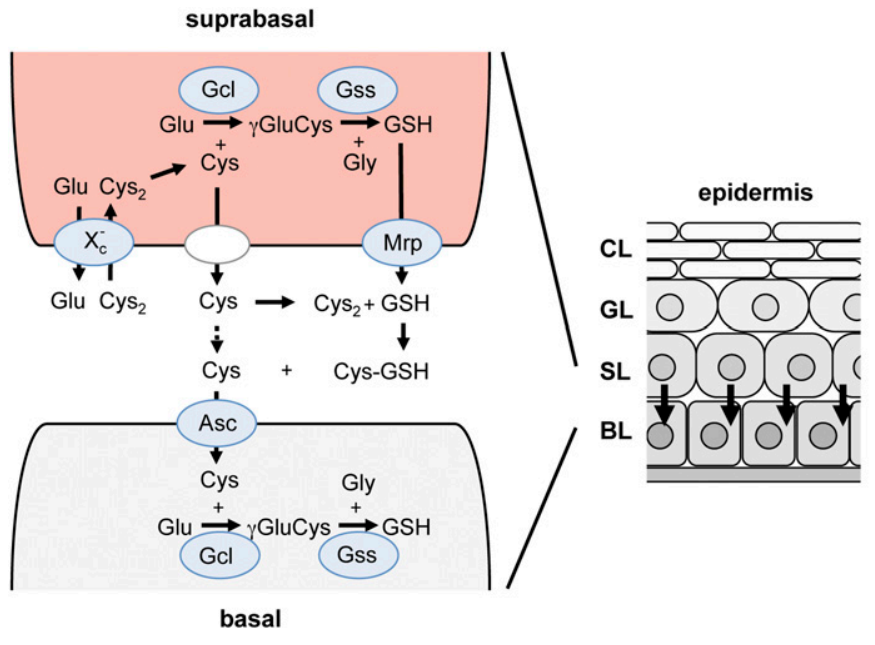

Figure 6. Model describing biosynthesis and recycling of GSH in the epidermis. (Middle) Scheme of the murine epidermis with basal, spinous, granular, and cornified layers. Arrows indicate a postulated paracrine cytoprotection of basal keratinocytes by suprabasal keratinocytes. (Left) Paracrine action of GSH and cysteine (Cys) involving suprabasal and basal layers. GSH is synthesized in a two-step reaction catalyzed by the enzymes Gcl and Gss. Extracellular cystine $\left(\mathrm{Cys}_{2}\right)$, the oxidized (predominant extracellular) form of cysteine, is imported into suprabasal cells by the antiporter $\mathrm{x}_{\mathrm{c}}{ }^{-}$. Intracellular cystine is reduced to cysteine, which is the rate-limiting amino acid for GSH synthesis. Cysteine as well as GSH are subsequently exported by suprabasal keratinocytes. GSH export occurs via Mrp transporters. Extracellular GSH provides cysteine via a thiol ex-

change reaction with cystine. Extracellular cysteine can be imported directly by basal keratinocytes via the importer Asc to serve as substrate for GSH synthesis in basal keratinocytes. Genes activated by Nrf2 in keratinocytes are encircled and highlighted with blue. (Right) A suprabasal-to-basal gradient of Nrf2 and its target genes coding for ROS-detoxifying enzymes and antioxidant proteins (in red) control UVB-induced apoptosis in the murine epidermis, resulting in an antagonistic basal-to-suprabasal gradient of apoptosis (in blue).

Most of the genes involved in this pathway are likely to be direct targets of Nrf2, since they were also up-regulated in primary keratinocytes of K5cre-caNrf2 mice (Supplemental Fig. S3C) and down-regulated in the epidermis of Nrf2 knockout mice (Supplemental Fig. S3D), and since they have been described as direct Nrf2 targets in other cells (Supplemental Table 1). The much higher expression of $x C T$ and Mrp3 (Fig. 5I), as well as of Nrf2, Gclc, Gclm, and Gsr (see Fig. 3E), in suprabasal versus basal keratinocytes of wild-type mice strongly suggests that a GSH- and cysteine-mediated paracrine cytoprotection of basal keratinocytes by suprabasal keratinocytes also exists in the epidermis of wild-type mice.

\section{Discussion}

We established a novel strategy to study the function of Nrf2 in vivo through expression of a caNrf2 mutant in keratinocytes. Increased Nrf2-mediated gene expression in all layers of the epidermis strongly reduced the rate of UVB-induced apoptosis. This is in line with the UVprotective effect of broccoli sprout extracts (DinkovaKostova et al. 2006), and with the enhanced UVB-induced apoptosis in ear skin keratinocytes of Nrf2 knockout mice (Kawachi et al. 2008). As the mechanism underlying the UVB-protective effect of caNrf2, we identified Nrf2mediated reduction of oxidative stress through enhanced expression of ROS-detoxifying enzymes and increased synthesis of GSH. Enhanced Nrf2 activity did not affect formation and repair of direct UVB-induced DNA lesions (CPDs). Consistent with this finding, CPD formation was also not altered in UVB-irradiated ear skin of Nrf2 knockout mice compared with wild-type controls (Kawachi et al. 2008). We even observed a decrease in the number of cells positive for $\mathrm{p} 53$ in caNrf2 transgenic mice, which is stabilized in response to DNA damage and ROS (Liu et al. 2008). Thus, Nrf2-mediated suppression of apoptosis is unlikely to result in an accumulation of keratinocytes with damaged DNA. Consistent with this finding, treatment of hairless mice with broccoli sprout extract containing sulforaphane, which among other effects activates Nrf2, reduced UVB-induced skin carcinogenesis (Dinkova-Kostova et al. 2006). Thus, activation of Nrf2 seems to be a useful strategy to reduce UVB-induced damage of keratinocytes. This is of major medical importance due to the strong increase in the incidence of epidermal skin cancer.

Interestingly, we noticed a basal-to-suprabasal gradient in UVB-induced apoptosis in the murine epidermis. This is surprising, since UVB is strongly absorbed by keratinocytes and should therefore target predominantly suprabasal keratinocytes (Anderson and Parrish 1981). On the other hand, this finding is biologically important, since prevention of apoptosis in suprabasal cells is essential for the maintenance of skin integrity after UVB irradiation. In contrast, damaged stem and transit-amplifying cells in the basal layer need to be eliminated to avoid malignant transformation. Our analysis in wild-type, caNrf2 transgenic, and Nrf2 knockout mice demonstrates that the remarkably selective protection of suprabasal, differentiated cells against apoptosis is achieved by the predominant expression of Nrf2 and its cytoprotective target genes suprabasally. The gradient of UVB-induced apoptosis is consistent with findings obtained with cultured murine and human keratinocytes, which showed enhanced UVB resistance upon differentiation (Chaturvedi et al. 2004; Sasaki et al. 2005; Mandinova et al. 2008). It may be that Nrf2 induces keratinocyte differentiation, but the normal differentiation pattern of keratinocytes that we observed in K10caNrf2 transgenic mice (see Supplemental Fig. S1F,G) does not support this hypothesis. Differentiation may also be affected by the levels of ROS. 
However, the normal differentiation of keratinocytes in mice overexpressing or lacking the ROS-detoxifying enzyme peroxiredoxin 6 in keratinocytes also argues against this possibility (Kumin et al. 2006, 2007). On the other hand, the enhanced UVB resistance of cultured keratinocytes upon differentiation may result from enhanced expression/activity of Nrf2 under these conditions. It will be interesting to address this question and to determine if the increased UVB resistance of in vitro differentiated keratinocytes is reduced or even abolished in keratinocytes from Nrf2 knockout mice.

In human skin, UVB-induced apoptosis is very limited in basal keratinocytes (Supplemental Fig. S4A,B). This may be due to the higher absorption of UVB light by the thick human epidermis, resulting in reduced exposure of basal keratinocytes to UVB. However, basal keratinocytes of mouse tail skin were also susceptible to UVBinduced apoptosis, although the tail epidermis also comprises multiple layers. Therefore, the most likely explanation for the different finding obtained with human compared with mouse skin is the existence of additional protective mechanisms, such as pigmentation (Supplemental Fig. S4C; Agar and Young 2005). In addition, cytoprotection seems to be increased in the basal layer of human skin, as no significant difference between suprabasal and basal expression of NRF2, NQO1, GCLC, and GCLM and only a mild up-regulation of $S R X N$ and GSTA3 in suprabasal layers were observed in human epidermis $(N=4)$ (Supplemental Fig. S4E). However, a basal-to-suprabasal gradient of apoptosis was observed within the suprabasal epidermis (Supplemental Fig. S4A,B). Whether the intra-suprabasal gradient of apoptosis is controlled by an Nrf2-mediated cytoprotective gradient within the suprabasal layers remains to be determined. In favor of such a hypothesis are results from in vitro experiments showing that progression of human keratinocyte differentiation results in increased activities of GST, GPX (GSH peroxidase), GSR, and total SOD. As mentioned above, this was accompanied by enhanced resistance to oxidative stress and reduced UVB-induced apoptosis of differentiated keratinocytes (Vessey et al. 1995; Sasaki et al. 2005; Mandinova et al. 2008).

Finally, our results demonstrate that Nrf2-mediated protection from UVB cytotoxicity in the murine epidermis is not only cell-autonomous. It has been shown in vitro that astrocytes protect neurons from ROS damage by secretion of cysteine and GSH (Desagher et al. 1996; Wang and Cynader 2000; Shih et al. 2003). Using biochemical and genetic approaches, we provide strong evidence for the existence of a similar paracrine mechanism in the epidermis of K10caNrf2 mice in vivo and most likely also in wild-type mice. The model that we propose is summarized in Figure 6. It is based on (1) the observed protective effect of caNrf2-expressing suprabasal keratinocytes for the underlying basal cells; (2) the dependency of this protection on GSH synthesis; (3) the up-regulation of genes involved in GSH production, secretion, and recycling by Nrf2 in keratinoctyes in vivo and in vitro; and (4) the predominant expression of $x C T$ and Mrp3 by suprabasal keratinocytes and of Asct 1 by basal keratinocytes. In addition, a first experiment with primary keratinocytes from wild-type and K5cre-caNrf2 transgenic mice revealed that mouse keratinocytes can indeed take up ${ }^{14} \mathrm{C}$-cystine, and that the import is enhanced by caNrf2 (data not shown).

While the role of GSH in the protection of basal cells was confirmed by the loss of the protection in a Gclmdeficient background, we cannot fully exclude the possibility that another paracrine-acting factor stimulates GSH production in basal keratinocytes. A potential candidate is Notch-1, which was shown recently to protect keratinocytes from UVB cytotoxicity (Mandinova et al. 2008). However, expression of the Notch-1 target genes Hes1, p21, $\Delta N p 63$, and Hey1 was not up-regulated in caNrf2 transgenic mice (data not shown), indicating that Notch-1 is not involved. This further strengthens our hypothesis that the paracrine-acting factor is the lowmolecular-weight antioxidant GSH and its precursor, cysteine.

The control of basal keratinocyte survival by suprabasal keratinocytes is likely to be of major biological importance. Under normal conditions, this mechanism could be required to maintain the redox balance and survival of basal keratinocytes, which express low levels of cytoprotective Nrf2 target genes. Upon UVB-induced damage of suprabasal keratinocytes, their protective function is reduced, as demonstrated by the inhibition of cystine import by keratinocytes in response to UVB irradiation and subsequent GSH depletion ( $\mathrm{Zhu}$ and Bowden 2004). Concomitantly, secretion of GSH and cysteine by suprabasal cells is diminished, resulting in apoptosis of basal keratinocytes. These findings highlight the importance of paracrine interactions and unravel a novel function of antioxidants in the cross-talk between cells at different stages of differentiation within a single tissue.

\section{Materials and methods}

\section{Generation of mice transgenic for caNrf2}

The cDNA encoding caNrf2 was amplified by PCR from cDNA of mouse skin wounds using oligonucleotides 5 '-TCCCCCCGG GCCACCATGCAGCACATCCAG-3' and 5'-CGGGATCCATC CTCCCGAAC-3', subcloned, and completely sequenced. It encodes amino acids 89-597 of the murine Nrf2 protein (NCBI NP_035032) (Fig. 1A). Transgenic mice were generated by pronucleus injection (FVB/N background for $\beta A C T c a N r f 2$, and B6D2F1 background for K10caNrf2). Genotyping of founder mice was performed by PCR with the following primers: for BACTcaNrf2, 5' -CGGCTCAGCACCTTGTAT-3' (down) and 5'-CT TATTCCAAGCGGCTTCGGC-3' (up); for K10caNrf2, the same down and 5' -CGGAATTCAGCTTGGTTCCCG-3' (up). All animal experiments were approved by the local veterinary authorities of Zurich, Switzerland (Kantonales Veterinäramt Zürich).

\section{Human skin biopsies}

Biopsies from abdominal human skin from four different female patients were provided by Dr. Gertrude Beer and Dr. Reinhard Dummer (University of Zurich) in the context of the Biobank project (approved by the local and cantonal institution review 
boards; EK no. 647). None of the patients claimed having taken a sunbath $1 \mathrm{wk}$ before the surgery, or to have excessive sunbathing habits (more than once a week on average).

Separation of basal and suprabasal keratinocytes, and of epidermis from dermis

For separation of basal and suprabasal keratinocytes, human abdominal skin or murine tail skin was incubated for $40 \mathrm{~min}$ at $37^{\circ} \mathrm{C}$ in $225 \mathrm{U} / \mathrm{mL} \alpha$-chymotrypsin A4 (Boehringer Mannheim) as described in Sasai et al. (1979), or in a less efficient $\alpha$-chymotrypsin Type IV (Sigma Aldrich). Afterward, the skin was washed in PBS, and the suprabasal layers were removed manually and snap-frozen in liquid nitrogen. Remaining basal keratinocytes were scraped off from the dermis in lysis buffer and were also snap-frozen. For separation of epidermis from dermis, murine back skin was incubated for $90 \mathrm{~min}$ at $37^{\circ} \mathrm{C}$ in $2.5 \mathrm{U} / \mathrm{mL}$ dispase (Invitrogen), and the epidermis was scraped off from the dermis and snap-frozen in liquid nitrogen.

\section{UVB irradiation}

UVB irradiation of mice was described previously (Kumin et al. 2007). Explants of human abdominal skin $\left(\sim 4 \mathrm{~cm}^{2}\right)$ were irradiated within $1 \mathrm{~d}$ post-operation with $100 \mathrm{~mJ} / \mathrm{cm}^{2} \mathrm{UVB}$ and were cultured together with control explants for $24 \mathrm{~h}$ in DMEM with high glucose (Sigma) containing $10 \mathrm{~mL} / \mathrm{L}$ penicillin/streptomycin (Sigma) and 3\% FCS (Bioconcept).

\section{Morphometric analysis of stratum corneum thickness}

Paraffin-embedded skin was cut from apical to basal to obtain $2-\mu \mathrm{m}$-thick sections. After H\&E staining, pictures were taken at $1000 \times$ magnification. Only areas where all layers of the stratum corneum were tightly together were morphometrically analyzed.

\section{Measurement of ROS and GSH levels, and of $\gamma$ GT activity}

Intracellular levels of ROS were determined in primary and immortalized keratinocytes using $\mathrm{H}_{2}$ DCF-DA (Invitrogen) as described previously (Durchdewald et al. 2007). For measurement of GSH levels, the epidermis was isolated from adult murine back skin. For this purpose, shaved back skin was incubated in $0.8 \%$ trypsin/PBS for $1 \mathrm{~h}$ at $37^{\circ} \mathrm{C}$, and the epidermis was scraped off and shaken for $1 \mathrm{~h}$ in defined K-SFM (Invitrogen) containing supplements, $10 \mathrm{ng} / \mathrm{mL}$ epidermal growth factor, and $10^{-10} \mathrm{M}$ cholera toxin (Sigma). Consequently, the suspension was filtered, centrifuged, and resuspended in 5\% 5-sulfosalicylic acid. After 15 min of incubation on ice and subsequent centrifugation, the supernatant was used for measurement of total GSH using the GSH assay kit (Sigma-Aldrich). The pellet was resuspended in $0.1 \mathrm{M} \mathrm{NaOH}$, and protein measurement was performed using BCA assay.

$\gamma \mathrm{GT}$ activity was analyzed on cryosections of murine back skin as described (Rutenburg et al. 1969).

\section{Morphometry, replicate experiments, and statistical analyses}

Morphometrical analysis was performed using ImageJ 1.410 (NIH) or Openlab 3.1.5 (Improvision). RPA and qRT-PCR were performed with RNA pools from two to four animals from one litter and were repeated with independent samples. Statistical analyses were performed using Prism version 5.0 software (GraphPat Software). All data were analyzed using the nonparametric Mann-Whitney $U$-test for non-Gaussian distribution. Error bars represent standard deviation.

\section{Acknowledgments}

We thank Christiane Born-Berclaz and Nicole Hallschmid for excellent technical assistance; Dr. Christina Rycken, Claudia Defila, Ann-Helen Willrodt, and Jörg Renkawitz for help with the characterization of the transgenic mice and cell culture experiments; Drs. Francois Verrey (University of Zurich, Switzerland), Simone Camargo (University of Zurich, Switzerland), and Tamara Ramadan (ETH Zurich, Switzerland) for valuable help with the ${ }^{14} \mathrm{C}$ cystine experiment; Drs. Gertrude Beer and Reinhard Dummer (University of Zurich) for providing human skin samples; Dr. Dietrich Keppler (DKFZ, Heidelberg, Germanyl for the Mrp3 and Mrp4 antibodies; Dr. José L. Jorcano (CIEMAT, Madrid, Spain) for the K5-Cre transgenic mice; Dr. Timothy Dalton (University of Cincinnati, Cincinnati, OH) for Gclm knockout mice; Dr. Yuet Wai Kan (University of California at San Francisco, San Francisco, CA) for Nrf2 knockout mice; and Dr. Manfred Blessing (University of Leipzig, Germany) for the K10 promoter. This work was supported by the Swiss National Science Foundation (grant 3100A0-109340 to S.W.); the Promedica Foundation, Chur, Switzerland (to S.W.); and the CERIES award (to S.W.). M.S. was supported by an EMBO post-doctoral fellowship and U.a.d.K was supported by a Boehringer Ingelheim predoctoral fellowship.

\section{References}

Agar N, Young AR. 2005. Melanogenesis: A photoprotective response to DNA damage? Mutat Res 571: 121-132.

Anderson RR, Parrish JA. 1981. The optics of human skin. I Invest Dermatol 77: 13-19.

auf dem Keller U, Huber M, Beyer TA, Kumin A, Siemes C, Braun S, Bugnon P, Mitropoulos V, Johnson DA, Johnson JA, et al. 2006. Nrf transcription factors in keratinocytes are essential for skin tumor prevention but not for wound healing. Mol Cell Biol 26: 3773-3784.

Beyer TA, Auf dem Keller U, Braun S, Schafer M, Werner S. 2007. Roles and mechanisms of action of the Nrf2 transcription factor in skin morphogenesis, wound repair and skin cancer. Cell Death Differ 14: 1250-1254.

Bickers DR, Athar M. 2006. Oxidative stress in the pathogenesis of skin disease. J Invest Dermatol 126: 2565-2575.

Bowden GT. 2004. Prevention of non-melanoma skin cancer by targeting ultraviolet-B-light signalling. Nat Rev Cancer 4: 23-35.

Braun S, Hanselmann C, Gassmann MG, auf dem Keller U, Born-Berclaz C, Chan K, Kan YW, Werner S. 2002. Nrf2 transcription factor, a novel target of keratinocyte growth factor action which regulates gene expression and inflammation in the healing skin wound. Mol Cell Biol 22: 54925505.

Chaturvedi V, Qin JZ, Stennett L, Choubey D, Nickoloff BJ. 2004. Resistance to UV-induced apoptosis in human keratinocytes during accelerated senescence is associated with functional inactivation of p53. J Cell Physiol 198: 100-109.

Chiba M, Jimbow K. 1986. Expression of $\gamma$-glutamyl transpeptidase in normal and neoplastic epithelial cells of human skin: A marker for distinguishing malignant epithelial tumours. Br J Dermatol 114: 459-464.

Christensen HN. 1990. Role of amino acid transport and countertransport in nutrition and metabolism. Physiol Rev 70: 43-77.

Dalton TP, Chen Y, Schneider SN, Nebert DW, Shertzer HG. 2004. Genetically altered mice to evaluate glutathione homeostasis in health and disease. Free Radic Biol Med 37: 1511-1526. 
Desagher S, Glowinski J, Premont J. 1996. Astrocytes protect neurons from hydrogen peroxide toxicity. I Neurosci 16: 2553-2562.

De Young LM, Richards WL, Bonzelet W, Tsai LL, Boutwell RK. 1978. Localization and significance of $\gamma$-glutamyltranspeptidase in normal and neoplastic mouse skin. Cancer Res 38: 3697-3701.

Dickinson SE, Melton TF, Olson ER, Zhang I, Saboda K, Bowden GT. 2009. Inhibition of activator protein-1 by sulforaphane involves interaction with cysteine in the cFos DNA-binding domain: Implications for chemoprevention of UVB-induced skin cancer. Cancer Res 69: 7103-7110.

Dinkova-Kostova AT, Jenkins SN, Fahey JW, Ye L, Wehage SL, Liby KT, Stephenson KK, Wade KL, Talalay P. 2006. Protection against UV-light-induced skin carcinogenesis in SKH-1 high-risk mice by sulforaphane-containing broccoli sprout extracts. Cancer Lett 240: 243-252.

Durchdewald M, Beyer TA, Johnson DA, Johnson JA, Werner S, auf dem Keller U. 2007. Electrophilic chemicals but not UV irradiation or reactive oxygen species activate Nrf2 in keratinocytes in vitro and in vivo. I Invest Dermatol 127: 646-653.

Fimognari C, Hrelia P. 2007. Sulforaphane as a promising molecule for fighting cancer. Mutat Res 635: 90-104.

Gruber F, Mayer H, Lengauer B, Mlitz V, Sanders JM, Kadl A, Bilban M, de Martin R, Wagner O, Kensler TW, et al. 2010. NF-E2-related factor 2 regulates the stress response to UVA1-oxidized phospholipids in skin cells. FASEB J 24: 39-48.

Hanada K, Gange RW, Connor MJ. 1991. Effect of glutathione depletion on sunburn cell formation in the hairless mouse. I Invest Dermatol 96: 838-840.

Ichihashi M, Ueda M, Budiyanto A, Bito T, Oka M, Fukunaga M, Tsuru K, Horikawa T. 2003. UV-induced skin damage. Toxicology 189: 21-39.

Itoh K, Wakabayashi N, Katoh Y, Ishii T, Igarashi K, Engel JD, Yamamoto M. 1999. Keap1 represses nuclear activation of antioxidant responsive elements by Nrf2 through binding to the amino-terminal Neh2 domain. Genes \& Dev 13: 76-86.

Jiang SJ, Chu AW, Lu ZF, Pan MH, Che DF, Zhou XJ. 2007. Ultraviolet B-induced alterations of the skin barrier and epidermal calcium gradient. Exp Dermatol 16: 985-992.

Johnson DA, Andrews GK, Xu W, Johnson JA. 2002. Activation of the antioxidant response element in primary cortical neuronal cultures derived from transgenic reporter mice. I Neurochem 81: 1233-1241.

Kannan S, Jaiswal AK. 2006. Low and high dose UVB regulation of transcription factor NF-E2-related factor 2. Cancer Res 66: 8421-8429.

Kawachi Y, Xu X, Taguchi S, Sakurai H, Nakamura Y, Ishii Y, Fujisawa Y, Furuta J, Takahashi T, Itoh K, et al. 2008. Attenuation of UVB-induced sunburn reaction and oxidative DNA damage with no alterations in UVB-induced skin carcinogenesis in Nrf2 gene-deficient mice. J Invest Dermatol 128: $1773-1779$.

Kensler TW, Wakabayashi N, Biswal S. 2007. Cell survival responses to environmental stresses via the Keap1-Nrf2ARE pathway. Annu Rev Pharmacol Toxicol 47: 89-116.

Kinlough CL, Poland PA, Bruns JB, Hughey RP. 2005. $\gamma$-Glutamyltranspeptidase: Disulfide bridges, propeptide cleavage, and activation in the endoplasmic reticulum. Methods Enzymol 401: 426-449.

Kokot A, Metze D, Mouchet N, Galibert MD, Schiller M, Luger TA, Bohm M. 2009. $\alpha$-Melanocyte-stimulating hormone counteracts the suppressive effect of UVB on Nrf2 and Nrfdependent gene expression in human skin. Endocrinology 150: $3197-3206$.
Kulms D, Zeise E, Poppelmann B, Schwarz T. 2002. DNA damage, death receptor activation and reactive oxygen species contribute to ultraviolet radiation-induced apoptosis in an essential and independent way. Oncogene 21: 5844-5851.

Kumin A, Huber C, Rulicke T, Wolf E, Werner S. 2006. Peroxiredoxin 6 is a potent cytoprotective enzyme in the epidermis. Am J Pathol 169: 1194-1205.

Kumin A, Schafer M, Epp N, Bugnon P, Born-Berclaz C, Oxenius A, Klippel A, Bloch W, Werner S. 2007. Peroxiredoxin 6 is required for blood vessel integrity in wounded skin. J Cell Biol 179: 747-760.

Lee DF, Kuo HP, Liu M, Chou CK, Xia W, Du Y, Shen J, Chen CT, Huo L, Hsu MC, et al. 2009. KEAP1 E3 ligase-mediated downregulation of NF-кB signaling by targeting IKK $\beta$. Mol Cell 36: 131-140.

Li Q, Tsuji H, Kato Y, Sai Y, Kubo Y, Tsuji A. 2006. Characterization of the transdermal transport of flurbiprofen and indomethacin. J Control Release 110: 542-556.

Liu B, Chen Y, St Clair DK. 2008. ROS and p53: A versatile partnership. Free Radic Biol Med 44: 1529-1535.

Lo M, Wang YZ, Gout PW. 2008. The $\mathrm{x}_{\mathrm{c}}{ }^{-}$cystine/glutamate antiporter: A potential target for therapy of cancer and other diseases. J Cell Physiol 215: 593-602.

Lu SC. 2009. Regulation of glutathione synthesis. Mol Aspects Med 30: 42-59.

Mandinova A, Lefort K, Tommasi di Vignano A, Stonely W, Ostano P, Chiorino G, Iwaki H, Nakanishi J, Dotto GP. 2008. The FoxO3a gene is a key negative target of canonical Notch signalling in the keratinocyte UVB response. EMBO /27: 1243-1254.

Marrot L, Jones C, Perez P, Meunier JR. 2008. The significance of Nrf2 pathway in (photo)-oxidative stress response in melanocytes and keratinocytes of the human epidermis. Pigment Cell Melanoma Res 21: 79-88.

Ramirez A, Page A, Gandarillas A, Zanet J, Pibre S, Vidal M, Tusell L, Genesca A, Whitaker DA, Melton DW, et al. 2004. A keratin K5Cre transgenic line appropriate for tissuespecific or generalized Cre-mediated recombination. Genesis 39: $52-57$.

Rutenburg AM, Kim H, Fischbein JW, Hanker JS, Wasserkrug HL, Seligman AM. 1969. Histochemical and ultrastructural demonstration of $\gamma$-glutamyl transpeptidase activity. $J$ Histochem Cytochem 17: 517-526.

Sasai Y, Kawamura K, Namba K. 1979. The separation of basal and differentiating cells from human epidermis for DNA cytofluorometry. Histochemistry 63: 265-272.

Sasaki H, Itoh T, Akamatsu H, Okamoto H, Horio T. 2005. Effects of calcium concentration on the SOD activity and UVB-induced cytotoxicity in cultured human keratinocytes. Photodermatol Photoimmunol Photomed 21: 9-14.

Shih AY, Johnson DA, Wong G, Kraft AD, Jiang L, Erb H, Johnson JA, Murphy TH. 2003. Coordinate regulation of glutathione biosynthesis and release by Nrf2-expressing glia potently protects neurons from oxidative stress. I Neurosci 23: 3394-3406.

Song HJ, Poy G, Darwiche N, Lichti U, Kuroki T, Steinert PM, Kartasova T. 1999. Mouse Sprr2 genes: A clustered family of genes showing differential expression in epithelial tissues. Genomics 55: 28-42.

Soriano FX, Leveille F, Papadia S, Higgins LG, Varley J, Baxter P, Hayes JD, Hardingham GE. 2008. Induction of sulfiredoxin expression and reduction of peroxiredoxin hyperoxidation by the neuroprotective $\mathrm{Nrf} 2$ activator $3 \mathrm{H}-1$,2-dithiole-3-thione. I Neurochem 107: 533-543.

Toohey JI. 1975. Sulfhydryl dependence in primary explant hematopoietic cells. Inhibition of growth in vitro with vitamin B12 compounds. Proc Natl Acad Sci 72: 73-77. 


\section{Schäfer et al.}

Tyrrell RM, Pidoux M. 1988. Correlation between endogenous glutathione content and sensitivity of cultured human skin cells to radiation at defined wavelengths in the solar ultraviolet range. Photochem Photobiol 47: 405-412.

Verschooten L, Declercq L, Garmyn M. 2006. Adaptive response of the skin to UVB damage: Role of the p53 protein. Int $J$ Cosmet Sci 28: 1-7.

Vessey DA, Lee KH, Boyer TD. 1995. Differentiation-induced enhancement of the ability of cultured human keratinocytes to suppress oxidative stress. J Invest Dermatol 104: 355-358.

Wakabayashi N, Itoh K, Wakabayashi J, Motohashi H, Noda S, Takahashi S, Imakado S, Kotsuji T, Otsuka F, Roop DR, et al. 2003. Keap1-null mutation leads to postnatal lethality due to constitutive Nrf2 activation. Nat Genet 35: 238-245.

Wang XF, Cynader MS. 2000. Astrocytes provide cysteine to neurons by releasing glutathione. I Neurochem 74: 14341442.

Wasserman WW, Fahl WE. 1997. Functional antioxidant responsive elements. Proc Natl Acad Sci 94: 5361-5366.

Werner S, Weinberg W, Liao X, Peters KG, Blessing M, Yuspa SH, Weiner RL, Williams LT. 1993. Targeted expression of a dominant-negative FGF receptor mutant in the epidermis of transgenic mice reveals a role of FGF in keratinocyte organization and differentiation. EMBO J 12: 2635-2643.

Yang Y, Dieter MZ, Chen Y, Shertzer HG, Nebert DW, Dalton TP. 2002. Initial characterization of the glutamate-cysteine ligase modifier subunit $\mathrm{Gclm}^{-1-}$ knockout mouse. Novel model system for a severely compromised oxidative stress response. J Biol Chem 277: 49446-49452.

Young AR. 1987. The sunburn cell. Photodermatol 4: 127-134.

Zhu M, Bowden GT. 2004. Molecular mechanism(s) for UV-B irradiation-induced glutathione depletion in cultured human keratinocytes. Photochem Photobiol 80: 191-196. 


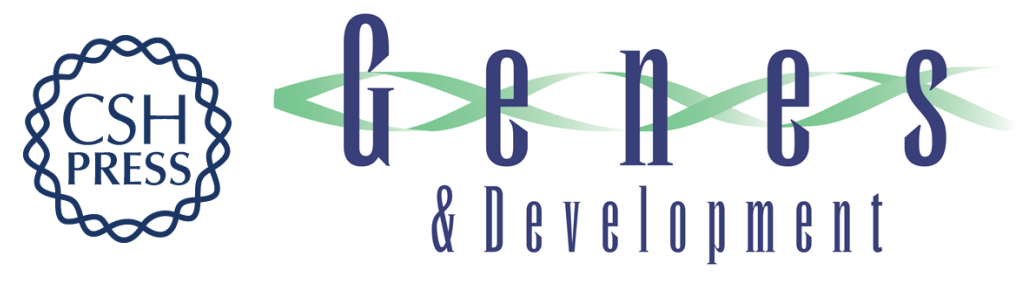

\section{Nrf2 establishes a glutathione-mediated gradient of UVB cytoprotection in the epidermis}

Matthias Schäfer, Sabine Dütsch, Ulrich auf dem Keller, et al.

Genes Dev. 2010, 24:

Access the most recent version at doi:10.1101/gad.568810

Supplemental http://genesdev.cshlp.org/content/suppl/2010/05/05/24.10.1045.DC1
Material

References This article cites 56 articles, 12 of which can be accessed free at:

http://genesdev.cshlp.org/content/24/10/1045.full.html\#ref-list-1

License

Email Alerting

Receive free email alerts when new articles cite this article - sign up in the box at the top

Service

right corner of the article or click here.

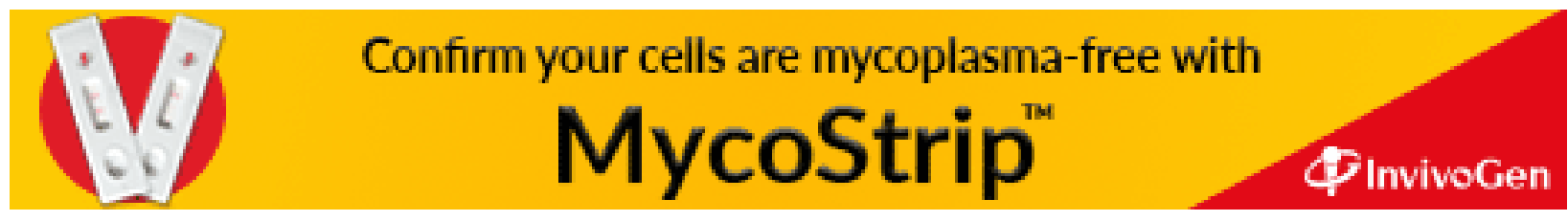

